\title{
Realization of High Aspect Ratio and Surface Defects in YZO Nanorods via Controlling Seed Layer Thickness
}

\author{
Narinder Kaur', Sejoon Lee ${ }^{1,2}$, and Deuk Young Kim ${ }^{1,2}$ \\ ${ }^{1}$ Quantum-Functional Semiconductor Research Center, Dongguk University-Seoul \\ Seoul 04623, Korea \\ narinderk@dongguk.edu \\ ${ }^{2}$ Department of Semiconductor Science, Dongguk University-Seoul \\ Seoul 04623, Korea \\ dykim@dongguk.edu
}

\begin{abstract}
High-aspect-ratio Y-doped $\mathrm{ZnO}$ (YZO) nanorods were grown vertically on $\mathrm{YZO}$-seeded $\mathrm{Si}$ substrates by using a hydrothermal method. The aspect ratios of YZO nanorods were altered by controlling the thicknesses of as-grown and $600{ }^{\circ} \mathrm{C}$-annealed YZO seed layers. The highest aspect ratio of YZO nanorods was observed to be $\sim 49.3$ by controlling the growth and in-situ etching process of $600{ }^{\circ} \mathrm{C}$-annealed YZO seed layers. Well-aligned YZO nanorods showed a single crystalline behavior corresponding to the preferential (002) orientation of the hexagonal wurtzite structure. PL and XPS results revealed that the surface defects were significantly increased with an increase of aspect ratio of YZO nanorods grown on the thicker as-grown and $600{ }^{\circ} \mathrm{C}$-annealed seed layers.
\end{abstract}

Keywords: YZO Nanorods, Seed Layers, Thickness, Aspect Ratio, Surface Defects.

\section{Introduction}

Various metal-oxides nanostructures like nanorods, nanowires, nanotubes, nanotrees, nanocombs, and nanoparticles have been paying attention in the technological world because of their great potential for magnetic, electronic, optical, and photocatalytic applications [1]. Among the metal oxides (e.g., $\mathrm{TiO}_{2}, \mathrm{ZnO}, \mathrm{Fe}_{2} \mathrm{O}_{3} / \mathrm{Fe}_{3} \mathrm{O}_{4}, \mathrm{Bi}_{2} \mathrm{O}_{3}, \mathrm{CeO}_{2}, \mathrm{MoO}_{2}, \mathrm{WO}_{3}$ ), $\mathrm{ZnO}$ is one of the most attractive materials owing to its unique physical properties such as a wide direct bandgap $(\sim 3.37 \mathrm{eV})$, a large exciton binding energy $(\sim 60 \mathrm{meV})$, high transparency in the visible and infrared wavelength regions, and low cost [2]. To date, several efforts have been made to realize the one-dimensional (1-D) $\mathrm{ZnO}$ nanostructures, e.g., nanorods and nanowires, for the integration of novel device $[3,4]$. In the direction of these efforts, the growth factors such as an aspect ratio, a density and an alignment have been effectively controlled via a number of parameters such as growth time, growth temperature, $\mathrm{pH}$ value, seed layer and the dopant concentration. Song et al. [5] investigated the effect of seed layer on the growth rate, diameter, density and the surface area of $\mathrm{ZnO}$ nanorods. Zhao et al. [6] reported that the nucleation mechanisms of vertically aligned $\mathrm{ZnO}$ nanorods were dependent on the Au catalyst. In addition to the growth factors, the surface defects (e.g., oxygen vacancies and oxygen interstitials) also signified a profound effect on the improvement of the photocatalytic activities of $\mathrm{ZnO}$ nanorods. Researchers found that the surface defects like $\mathrm{OH}$ - ions adsorb onto (0001)-Zn surface due to its surface positive charge and react with holes to produce reactive species. The large number of electron and holes exit on the active sites of $\mathrm{ZnO}$ nanostructures due to its one-dimensional anisotropy behavior, which then increase the photocatalytic response towards various kinds of persistent organic pollutants [7]. Thus, the combined effect of the surface defects and the growth factors such as aspect-ratio play a vital role to improve the photocatalytic activity of ZnO nanorods.

In the meantime, the modification of $\mathrm{ZnO}$ nanostructures has been demonstrated with addition of extra foreign impurities like yttrium (Y) in $\mathrm{ZnO}$ lattices because the presence of yttrium (Y) enhanced the performance as well as the stability of devices [8]. Recently, Das et al. [9] observed that the efficiency of YZO based organic solar cell device was improved by $30 \%$ as compared to un-doped $\mathrm{ZnO}$ based devices. In addition, modified nanostructures of YZO showed the higher sensing response towards different gases [10]. Keeping the above-mentioned perspectives in mind, the study of the modification of the aspect-ratio and the surface defects in YZO nanorods is very essential step for the establishment of the 
rare-earth material based future technology. To move a closer step in this direction, the understandings of the growth parameters in YZO nanorods and their effect on the realization of the high aspect-ratio and surface defects is highly desirable. However, on this way, the effects of seed-layers' thicknesses on the growth and the characterization of YZO nanorods have not studied well. In this article, we demonstrate the growth of high-aspect-ratio and surface defects in YZO nanorods by modulating the grains of thickness-dependent as-grown and $600{ }^{\circ} \mathrm{C}$-annealed YZO seeded-Si-substrates. The effects of YZO seed layer thickness were distinguished in terms of the microstructure analysis, chemical bonding, structural, and optical properties of YZO nanorods.

\section{Experiment}

All chemicals of analytical grade were used without further purification. The precursor solution for the growth of YZO (Y: 2 at.\%) seed layers was prepared by the dissolving of zinc acetate dehydrate and yttrium acetate hydrate in the solvent of 2-methoxyethanol and consequently stirred at $60^{\circ} \mathrm{C}$ for $2 \mathrm{~h}$. To remove the turbidity and precipitates, a stabilizing agent, "monoethanolamine" was added dropwise to the prepared sol. Finally, the aged sol was spin coated on the ultrasoniccleaned Si substrates and subsequent dried in oven at $300{ }^{\circ} \mathrm{C}$ for $10 \mathrm{~min}$ to evaporate the solvent and organic residues. To manipulate the thicknesses of YZO seed layers, the spin-coating and drying processes were repeated by 2, 5, and 10 times. Thereafter, YZO seed layers were annealed in vacuum at $600{ }^{\circ} \mathrm{C}$ for $3 \mathrm{~min}$ by using a rapid-thermal-annealing system. Finally, as-grown $(2 \mathrm{~S} 1,5 \mathrm{~S} 1$, and $10 \mathrm{~S} 1)$ and $600{ }^{\circ} \mathrm{C}$-annealed $(2 \mathrm{~S} 6,5 \mathrm{~S} 6$, and 10S6) $\mathrm{YZO}$ seed layers corresponding to 2,5 , and 10 times of spin-coating processes were chosen for the further growth of YZO nanorods. In order to prepare the YZO $(0.1 \mathrm{M})$ solution for the growth of nanorods, the precursors of zinc nitrate hexahydrate, yttrium acetate hydrate, and hexamethylenetetramine were mixed in the $35 \mathrm{ml}$ of DI water. To perform the hydrothermal reactions, the autoclaves containing samples were kept on the hot plate at $130{ }^{\circ} \mathrm{C}$ for $4 \mathrm{~h}$. After completing the reaction time, samples were taken away from the autoclaves and washed with the DI water several times to remove the surface contaminants and nitrate ions. The surface morphologies and the cross-section views of YZO seed layers and nanorods were examined by the Field Emission-Scanning Electron Microscopy (Hitachi-S-4700). The crystalline behavior of YZO nanorods were investigated by using X-ray Diffractometer (Rigaku-Ultima-IV). Near-band-emission and deep-level-emission for YZO nanorods were analyzed with the help of room-temperature Photoluminescence Spectroscopy (PL) using an excitation source of He-Cd laser. The chemical bonding states of oxygen $(\mathrm{O})$ species in YZO nanorods were evaluated through X-ray Photoelectron Spectroscopy (PH15000 Versa Probe II).

\section{Results and Discussion}

Figs. 1 (a) - (b) illustrate the surfaces and the cross-sectional (inset) FE-SEM images of the as-grown (2S1, 5S1, 10S1) and $600{ }^{\circ} \mathrm{C}$-annealed (2S6, 5S6, 10S6) YZO seed layers corresponding to the film thicknesses of $\sim 200 \mathrm{~nm}, \sim 350 \mathrm{~nm}$, and $\sim 650 \mathrm{~nm}$, respectively. The thicknesses of as-grown and $600{ }^{\circ} \mathrm{C}$-annealed $\mathrm{YZO}$ seed layers were increased with an increase of number of spin-coatings sequence times (Fig. 1 (c)). It is observed from the surface analysis that the grains of as-grown and $600{ }^{\circ} \mathrm{C}$-annealed $\mathrm{YZO}$ seed layers were uniformly distributed throughout the film surfaces and seemed to be the closely-packed structures. The average grain sizes (Fig. 1 (d)) of as-grown $(2 \mathrm{~S} 1 \rightarrow 10 \mathrm{~S} 1)$ YZO seed layers were increased from $\sim 16$ to $\sim 25 \mathrm{~nm}$ as the thickness of the films increased from $\sim 200$ to $\sim 650 \mathrm{~nm}$. However, the grains of 600 ${ }^{\circ} \mathrm{C}$-annealed YZO seed layers $(2 \mathrm{~S} 6(\sim 30 \mathrm{~nm}) \rightarrow 10 \mathrm{~S} 6(\sim 40 \mathrm{~nm}))$ were observed to be bigger than the grains of as-grown YZO seed layers $(2 \mathrm{~S} 1(\sim 16 \mathrm{~nm}) \rightarrow 10 \mathrm{~S} 1(\sim 25 \mathrm{~nm}))$. The increase in the grain sizes of $600^{\circ} \mathrm{C}$-annealed YZO seed layers could be understood by an Ostwald ripening mechanism [11]. Ostwald ripening is a thermal energy dependent process and it becomes more significant as the annealing temperature of film increased. In our case, during the annealing process, the sufficient energy provides to start the growth of small seed grains of YZO films. The formation of small seed grains reduces the super saturation and initiates to start the coalescence process. This coalescence among the small grains develops the inter-grain connection, which will facilitate the formation of dense and bigger grains of annealed-YZO seed layer. Therefore, the coalescence process is dominated by Ostwald ripening mechanism in which the bigger grains are found to be occurred at the expense of smaller particles [12]. On the other side, as could be seen in the Fig. 1 (d), the grain sizes of both YZO seed layers (as grown and $600^{\circ} \mathrm{C}$-annealed) were increased by increasing the thicknesses (i.e., number of spin coating times) of seed layers. It is generally perceived that grain growth of films with varying thickness depends upon the lattice mismatch and strain induced by the substrate on which the films of various thicknesses have been grown. For the present case, it is anticipated that when the number of spin coatings layers increases, high density of nuclei leads to 
combine with each other and then, reduces the surface strain energy as well as the free energy of grain boundaries. This may be the possible cause for the increase growth of grains in YZO seed layers having various thicknesses [13].

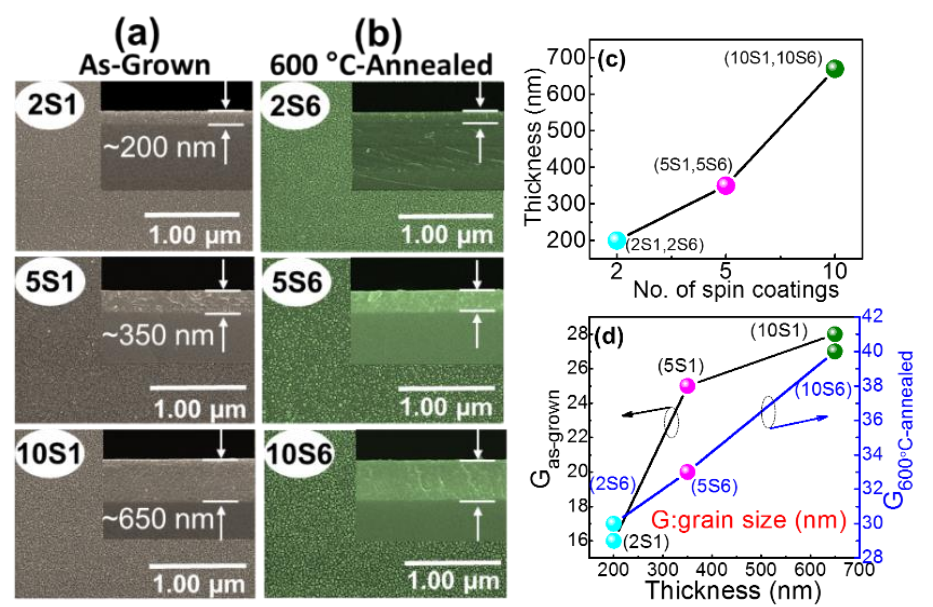

Fig. 1: Surface and cross-sectional (inset) FE-SEM images of (a) as-grown $(2 \mathrm{~S} 1 \rightarrow 10 \mathrm{~S} 1)$ and (b) $600{ }^{\circ} \mathrm{C}$-annealed $(2 \mathrm{~S} 6 \rightarrow 10 \mathrm{~S} 6) \mathrm{YZO}$ seed layers of different thicknesses, (c) Variations of seed layer's thicknesses vs. number of spin coatings layers, and (d) Grain sizes of as-grown and $600{ }^{\circ} \mathrm{C}$-annealed $\mathrm{YZO}$ seed layers as a function of film thicknesses.

Figs. 2 (a) - (b) display the surfaces and the cross-sectional (inset) FE-SEM images of YZO nanorods grown on the asgrown and $600^{\circ} \mathrm{C}$-annealed $\mathrm{YZO}$ seed layers of various thicknesses. To understand the sole effect of thickness-dependent as-grown and $600^{\circ} \mathrm{C}$-annealed $\mathrm{YZO}$ seed layers on the growth of $\mathrm{YZO}$ nanorods, the growth parameters such as mole concentration of reactants, growth time, growth temperature, and $\mathrm{pH}$ value were remained constant while performing the hydrothermal reactions. The hydrothermal mechanism for the growth of nanorods can be understood by the following chemical reactions performed in the autoclaves [14]:

$$
\begin{gathered}
\left(\mathrm{CH}_{2}\right)_{6} \mathrm{~N}_{4}+6 \mathrm{H}_{2} \mathrm{O} \rightarrow 6 \mathrm{HCHO}+4 \mathrm{NH}_{3} \\
\mathrm{NH}_{3}+\mathrm{H}_{2} \mathrm{O} \rightarrow \mathrm{NH}_{4}^{+}+\mathrm{OH}^{-} \\
\mathrm{Zn}^{2+}+2 \mathrm{OH}^{-} \rightarrow \mathrm{Zn}(\mathrm{OH})_{2} \rightarrow \mathrm{ZnO}+\mathrm{H}_{2} \mathrm{O}
\end{gathered}
$$

During the hydrothermal processes, the HMT acted as a buffer agent to hydrolyze and release $\mathrm{NH}_{3}$ for providing $\mathrm{OH}^{-}$ions. While, zinc nitrate precursor was used for providing $\mathrm{Zn}^{2+}$ ions. The reactions between $\mathrm{Zn}^{2+}$ and $\mathrm{OH}^{-}$ions would be continued until the intermediate product $\left(\mathrm{Zn}(\mathrm{OH})_{2}\right)$ reduced to the final product of $\mathrm{ZnO}$ in the aqueous solution. The hydrothermal processes were continued for $4 \mathrm{~h}$ in order to attain the vertical growth of YZO nanorods on the thicknessvariant-YZO seed layers. From the Figs. 2 (a)-(b), the nanorods growth along the c-axis with hexagonal morphology indicated that $\mathrm{Y}$-dopant has well placed in the lattice sites of $\mathrm{ZnO}$ and maintained the wurtzite structure of the host material. By taking the surface and cross-section analysis into account, the average diameter, average length and the aspect-ratio were estimated for the $\mathrm{YZO}$ nanorods grown on thickness-dependent as-grown and $600^{\circ} \mathrm{C}$-annealed $\mathrm{YZO}$ seed layers. It is observed form the Fig. 2 (c), the average diameter $(\sim 216 \mathrm{~nm})$ of YZO nanorods showed negligible changes as the thickness of as-grown YZO seed layer increased from $\sim 200$ to $\sim 350 \mathrm{~nm}$. Generally, the diameter of nanorods is controlled by the grain size of underlined seed layer. The grain sizes of as-grown YZO seed layers provide the effective nucleation sites for the growth of YZO nanorods. Thus, an insignificant change in the average diameter of YZO nanorods ( $2 \mathrm{R} 1$ and $5 \mathrm{R} 1$ ) might be expected due to the little variation in the grain sizes of as-grown YZO seed layers (2S1 and 5S1). However, the average length of YZO nanorods $(2.7 \mu \mathrm{m}(2 \mathrm{R} 1) \rightarrow 2.75 \mu \mathrm{m}(5 \mathrm{R} 1))$ (Fig. 2 (c)) was slightly increased which resulted to cause a small increase in the aspect-ratio $(12.5 \rightarrow 12.7)$ (Fig. 2 (e)) of YZO nanorods. A slight enhancement in the vertical growth rather than lateral growth might be ascribed to the improvement in the lowest surface energy density of (002) plane. On the other side, when the thickness of as-grown seed layer was increased up to $\sim 650 \mathrm{~nm}$, the average 
diameter of YZO nanorods was reduced from 216 to $150 \mathrm{~nm}$, and the length of YZO nanorods was increased from 2.75 to $3.5 \mu \mathrm{m}$ (Fig. 2 (c)). As a result, the aspect-ratio was increased from 12.7 to 23.3 for YZO nanorods grown on $\sim 650 \mathrm{~nm}$-asgrown-YZO seed layer (Fig. 2 (e)). This behavior could be attributed to the competition growth mechanism between (0001) crystal plane and non-polar side planes. Due to the suppression of non-polar plane and the modulation of dense nanoparticles on thicker-as-grown-YZO seed layer, YZO nanorods were expected to grow vertically with the high degree of orientation along c-axis to the substrate. In order to achieve an improvement in the aspect-ratio, the changes in the average diameter and the average length of $\mathrm{YZO}$ nanorods were noticed on the thickness-variant $600{ }^{\circ} \mathrm{C}$-annealed-YZO seed layer. As we can see from the Fig. 2 (d), the average diameter of YZO nanorods (2R6 $\rightarrow 5 \mathrm{R} 6)$ was observed to be decreased from 150 to $75 \mathrm{~nm}$ with increasing thickness of $600{ }^{\circ} \mathrm{C}$-annealed seed layer $(2 \mathrm{~S} 6 \rightarrow 5 \mathrm{~S} 6)$. Correspondingly, the value of aspect ratio of YZO nanorods grown on $600{ }^{\circ} \mathrm{C}$-annealed seed layers is increased from 25.3 to 33.3 due to the reduction in the diameter of nanorods (Fig. 2 (e)). Moreover, a further increase in aspect-ratio (49.3) of nanorods grown on thicker-annealed-seed layer $(\sim 650 \mathrm{~nm})$ was observed because of the increase in nanorods length from 2.5 to $3.7 \mu \mathrm{m}$. However, the diameter of YZO nanorods grown on the thicker-600 ${ }^{\circ} \mathrm{C}$-annealed seed layer showed negligible changes (Fig. 2 (d)). The aspect-ratio of YZO nanorods can be controlled by the growth and in-situ etching process of $600{ }^{\circ} \mathrm{C}$ annealed seed layers. In the case of thinner seed layer (2S6), most of the small grains can be etched off by the hydroxide ions $(\mathrm{OH}-)$ during the growth process and remained the big YZO grains, which will act as the nuclei for the growth of less dense YZO nanorods (2R6). Thus, it can be said that YZO nanorods (2R6) of higher diameter ( 150 nm) and lower density were expected to grow on the persisted seed grains which might be obtained through the etching of $\mathrm{OH}-$ ions. Moreover, Annealing at high temperature $\left(600{ }^{\circ} \mathrm{C}\right)$ will provide the more kinetic energy for the mobility of seed particles on YZO seed layer of different thickness. For the seed layer having lowest thickness (2S6), less nuclei sites with good lattice match between the seed layer nuclei and YZO nanorods are achieved which results to slower growth rate and lower density of YZO nanorods (2R6). After the etching of grains in the initial growth process, remaining YZO nuclei of bigger size will aggregate and become greater than that nuclei of thinner seed layer, resulting in the higher density of oriented nanorods (5R6) on the seed layer (5S6) of thickness $\sim 350 \mathrm{~nm}$. Finally, the growth speed of YZO nanorods with small nucleation densities is faster than nanorods with high nucleation densities. Thus, the length of YZO nanorods on the thicker seed layer $(\sim 650 \mathrm{~nm})$ was improved which led to increase the high-aspect-ratio (49.3) of YZO nanorods. Also, the bigger seed particles of thicker seed layer limit the migration of nuclei and lower the lattice stress caused by lattice mismatch between YZO seed layer and Si substrates. Therefore, more nuclei sites of thicker $600{ }^{\circ} \mathrm{C}$-annealed YZO seed layer provide the faster growth rate of dense YZO nanorods, which results to the increase of aspect-ratio. So, YZO nanorods with highaspect-ratio (49.3) can be achieved by the appropriate control of seed layer thickness and the annealing temperature. It can be concluded from the above analysis that the aspect-ratio of YZO nanorods are highly sensitive to the thicknesses and the annealing temperature of YZO seed layers.

(a) As-Grown
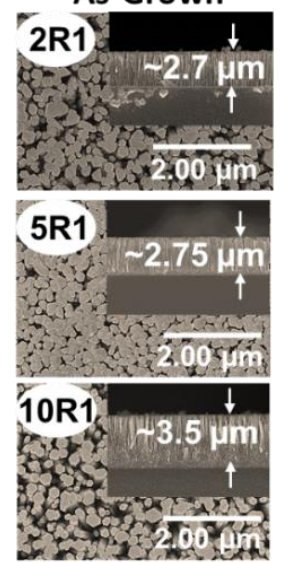

(b)

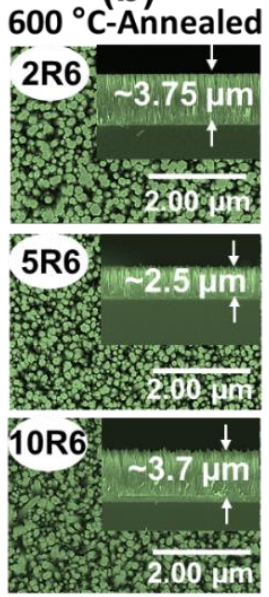

(c)

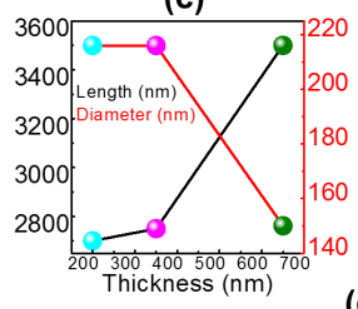

(e)
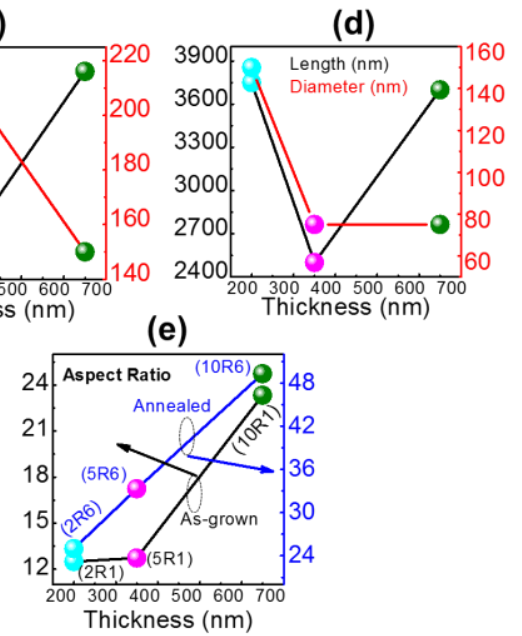

Fig. 2: Surface and cross-sectional (inset) FE-SEM images of YZO nanorods grown on (a) as-grown $(2 \mathrm{R} 1 \rightarrow 10 \mathrm{R} 1)$ and $(\mathrm{b}) 600^{\circ} \mathrm{C}$ annealed $(2 \mathrm{R} 6 \rightarrow 10 \mathrm{R} 6)$ YZO seed layers of various thicknesses. Changes in the average diameter and average length of as-grown (c) and $600{ }^{\circ} \mathrm{C}$-annealed (d) seeded-YZO nanorods, and (e) variation in the aspect-ratio of YZO nanorods with respect to various thicknesses. 
Figs. 3 (a) - (b) show the XRD patterns of YZO nanorods grown on the as-grown and $600{ }^{\circ} \mathrm{C}$-annealed YZO seed layers of different thicknesses. Regardless of the variation in aspect ratios, all YZO nanorods showed the single crystalline behavior corresponding to the preferential (002) orientation of the hexagonal wurtzite structure (JCPDS-36-1451). No extra peaks corresponding to the yttria or yttrium oxide phases were noticed in XRD patterns, which confirmed the formation of high quality YZO nanorods with a successful replacement of Y ions into the $\mathrm{Zn}$ sites. The crystallite sizes of YZO nanorods were evaluated from the Scherer's equation [8]

$$
D=0.94 \lambda /(\beta \cos \theta)
$$

where $\lambda$ is the $\mathrm{X}$-ray wavelength $(0.154 \mathrm{~nm}), \theta$ is the Bragg diffraction angle, and $\beta$ is the full width at half maximum (FWHM). With an increase of thickness of as-grown YZO seed layers (2S1 $\rightarrow 10 \mathrm{~S} 1)$, FWHM values were slightly increased $(174 \rightarrow 175)$ and the crystallite size of YZO nanorods were decreased $(49.94 \rightarrow 47.44)$. The similar behavior of increase in FWHM $(0.166 \rightarrow 0.199)$ and decrease in crystallite sizes $(50.10 \rightarrow 41.72)$ was noticed in the case of YZO nanorods $(2 \mathrm{R} 6 \rightarrow 10 \mathrm{R} 6)$ grown on the thickness-variant $600{ }^{\circ} \mathrm{C}$-annealed seed layers.
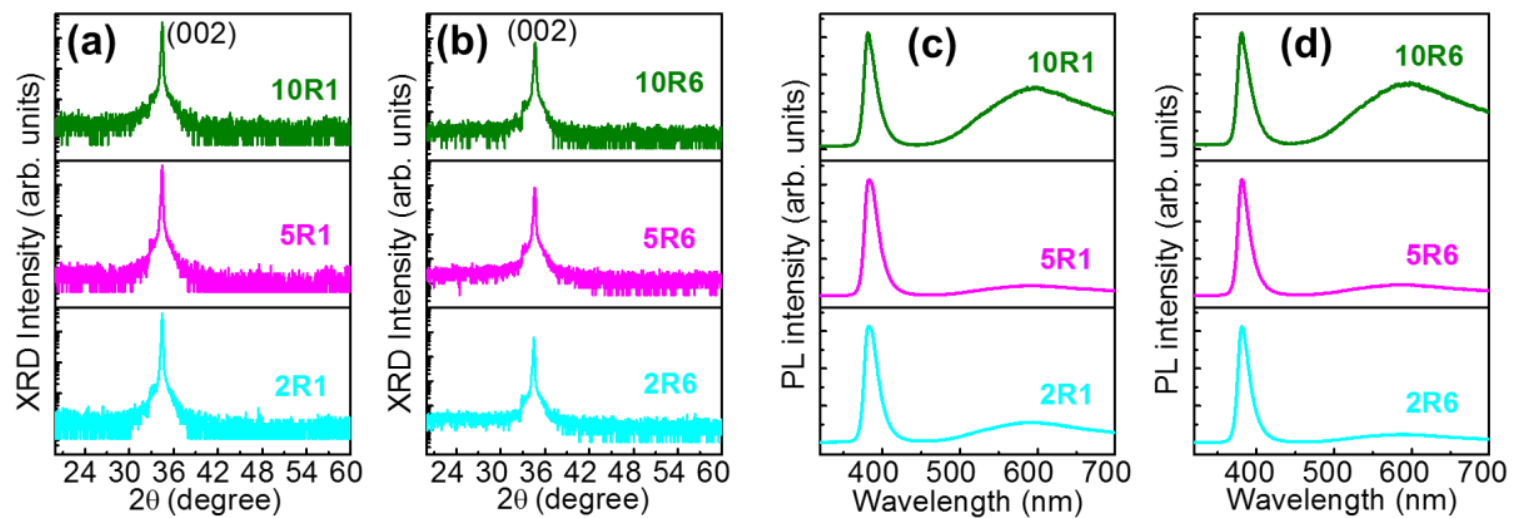

Fig. 3: X-ray diffraction pattern of YZO nanorods grown on (a) as-grown and (b) $600{ }^{\circ} \mathrm{C}$-annealed $\mathrm{YZO}$ seed layers having various thicknesses. PL spectra of YZO nanorods grown on thickness-variant as-grown (c) and $600{ }^{\circ} \mathrm{C}$-annealed (d) $\mathrm{YZO}$ seed layers.

Figs. 3 (c) - (d) show the room temperature PL spectra of YZO nanorods grown on the thickness-dependent as-grown and $600{ }^{\circ} \mathrm{C}$-annealed YZO seed layers. The PL spectra of all YZO nanorods consist of two distinct peaks, one is positioned at $\sim 381 \mathrm{~nm}$, that is, at the near band edge (NBE) emission, and other one centered at $\sim 593 \mathrm{~nm}$ in the wide range of visible region. The peak located at $\sim 381 \mathrm{~nm}$, assigned to free excitons $\left(\mathrm{FX}_{\mathrm{o}}\right)$ recombination corresponding to near band edge (NBE) emission, while other peak centered at $\sim 593 \mathrm{~nm}$ is related to the deep level emission (DLE) [15, 16]. It can be seen form the PL spectra that there were no noticeable changes occurred in the NBE emissions while DLE emissions of YZO nanorods were increased by increasing the thickness of as-grown $(2 \mathrm{R} 1 \rightarrow 10 \mathrm{R} 1)$ and $600{ }^{\circ} \mathrm{C}$-annealed $\mathrm{YZO}$ seed layers $(2 \mathrm{R} 6 \rightarrow 10 \mathrm{R} 6)$. The DLE in YZO nanorods is identified as the yellow emission emerging within the visible range of 500 to $700 \mathrm{~nm}$. According to the previous literature, the yellow emission is usually contributed by oxygen-related-defects such as oxygen vacancy and oxygen interstitials [17, 18]. Moreover, the Yellow emission can be attributed to the existence of hydroxyl group $(-\mathrm{OH})$ or $\mathrm{Zn}(\mathrm{OH})_{2}$ on the surface of $\mathrm{YZO}$ nanorods [19-21]. So, it is believed that the emission of yellow band may arise due to the oxygen-related-defects as well the presence of hydroxyl ions on the surface of YZO nanorods. Moreover, the defects tendency were appeared to greater in the case of YZO nanorods grown on the thicker as-grown (10R1) and annealed seed layers (10R6).

To clarify the oxygen-related-defects in YZO nanorods corresponding to the thickness-variant as-grown and $600{ }^{\circ} \mathrm{C}$ annealed YZO seed layers, XPS spectra of oxygen (O1s) species were taken, as shown in the Figs. 4 (a) - (b). The O1s spectra is fitted with Gaussian function and deconvoluted to three different oxygen peaks in YZO nanorods. The dominant peak (I) with low binding energy $(\sim 530 \mathrm{eV})$ was attributed to $\mathrm{O}^{2-}$ ions binding with nearest neighbor metal ions in host material $\mathrm{ZnO}$. The second peak (II) at the binding energy of $\sim 531 \mathrm{eV}$ was reported as the presence of $\mathrm{O}^{2-}$ ions in the 
oxygen deficiency regions within the matrix of $\mathrm{ZnO}$, which indicated the existence of oxygen vacancies. The third peak (III) with high binding energy $(\sim 532 \mathrm{eV})$ was identified as the presence of loosely bound oxygen (e.g., hydroxyl $(-\mathrm{OH})$ groups) on the surface of YZO nanorods. In the present case, the peaks (II \& III) related to the oxygen defects such as oxygen vacancies and hydroxyl ions were increased with respect to the YZO nanorods grown on the thicknessdependent as-grown $(2 \mathrm{R} 1 \rightarrow 10 \mathrm{R} 1)$ and $600^{\circ} \mathrm{C}$-annealed $(2 \mathrm{R} 6 \rightarrow 10 \mathrm{R} 6) \mathrm{YZO}$ seed layers. From the XPS observation, it is emphasized that the abundant surface oxygen vacancies or defects exit in the YZO nanorods grown on the thicker $(\sim 650 \mathrm{~nm})$ YZO-seed layers (as-grown and $600{ }^{\circ} \mathrm{C}$-annealed). The XPS result is in well agreement with the above mentioned PL result.
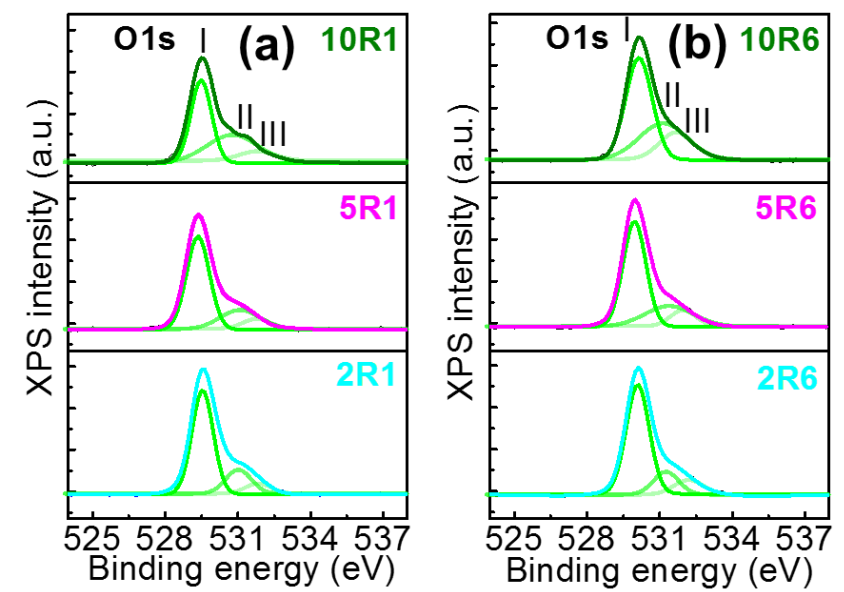

Fig. 4: Deconvolution of XPS O1s spectra of YZO nanorods on thickness-dependent as-grown (a) and $600{ }^{\circ} \mathrm{C}$-annealed (b) YZO seed layers.

In order to correlate the oxygen-related-defects with the aspect-ratio, variations in the aspect-ratio and DLE (deeplevel-emission) intensity of YZO nanorods were shown in the Figs. 5 (a) - (b) as a function of thickness-variant as-grown and $600^{\circ} \mathrm{C}$-annealed $\mathrm{YZO}$ seed layers.
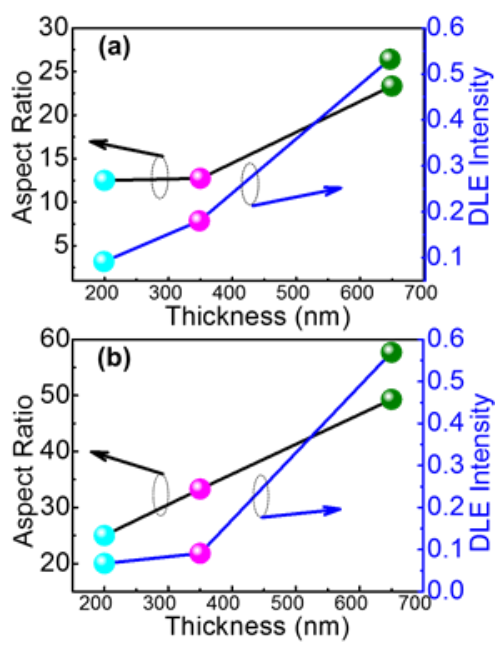

Fig. 5: Variations in the aspect ratio and DLE intensity of YZO nanorods grown on thickness-dependent as-grown (a) and $600{ }^{\circ} \mathrm{C}-$ annealed (b) YZO seed layers.

Fig. 5 (a) depicts that when the thickness of as-grown YZO seed layers $(\sim 200 \rightarrow \sim 650 \mathrm{~nm})$ increased, the aspect-ratio and deep-level emission intensity of YZO nanorods were also monotonically increased. Likewise, the aspect-ratio and deep-level emission intensity were reached to its maximum value for the $\mathrm{YZO}$ nanorods grown on the thicker-600 ${ }^{\circ} \mathrm{C}$ - 
annealed YZO seed layer (Fig. 5 (b)). These results demonstrate that the seed layer's thickness $(\sim 650 \mathrm{~nm})$ and subsequent annealing temperature $\left(600^{\circ} \mathrm{C}\right)$ have profound effect on the growth of high-aspect-ratio $(\sim 49.3)$ nanorods which exhibit the high level of surface defects.

\section{Conclusion}

We demonstrated the monitoring of high-aspect-ratio YZO nanorods by varying the thickness of as-grown and 600 ${ }^{\circ} \mathrm{C}$-annealed YZO seed layers on Si substrates. The highest aspect-ratio ( 49.3) of YZO nanorods was observed on the thicker- $600{ }^{\circ} \mathrm{C}$-annealed seed layer $(\sim 650 \mathrm{~nm})$ via modulating the seed grains through growth and in-situ etching process of seed layers. YZO nanorods on thicker- $600{ }^{\circ} \mathrm{C}$-annealed seed layer confirmed the single crystalline behavior corresponding to the preferential (002) orientation of the hexagonal wurtzite structure. Moreover, YZO nanorods grown on the thicker- $600{ }^{\circ} \mathrm{C}$-annealed $\mathrm{YZO}$ seed layer provides the more surface defects (e.g., oxygen vacancies and hydroxyl ions). The results of this study revealed that the appropriate control of thickness and annealing of seed layer has profound effect on the aspect ratio and surface defects of YZO nanorods, which will proposes the idea of using high-aspect-ratio YZO nanorods as a photocatalysts in the future work.

\section{Acknowledgement}

This research was supported by the National Research Foundation of Korea through the Basic Science Research Program (2016R1A6A1A03012877) funded by the Ministry of Education of the Korean government.

\section{References}

[1] Y. Gyu-Chul, W. Chunrui, and P. Won Il, "ZnO nanorods: synthesis, characterization and applications," Semiconductor Science and Technology, vol. 20, no. 4, pp. S22, 2005.

[2] K. S. Kim, H. Jeong, M. S. Jeong, and G. Y. Jung, "Polymer-Templated Hydrothermal Growth of Vertically Aligned Single-Crystal ZnO Nanorods and Morphological Transformations Using Structural Polarity," Advanced Functional Materials, vol. 20, no. 18, pp. 3055-3063, 2010.

[3] H. B. Lee, R. T. Ginting, S. T. Tan, C. H. Tan, A. Alshanableh, , H. F. Oleiwi, C. C. Yap, M. H. H. Jumali, and M. Yahaya, "Controlled Defects of Fluorine-incorporated $\mathrm{ZnO}$ Nanorods for Photovoltaic Enhancement," Scientific Reports, vol. 6, pp. 32645, 2016.

[4] A. Orlov, V. Ulianova, A. Zazerin, O. Bogdan, G. Pashkevich, and Y. Yakymenko, "Active elements on a basis of $\mathrm{ZnO}$ nanorods for energy harvesting devices," Radioelectronics and Communications Systems, vol. 59, no. 2, pp. 6065, 2016.

[5] J. Song, and S. Lim, "Effect of Seed Layer on the Growth of ZnO Nanorods," The Journal of Physical Chemistry C, vol. 111, no. 2, pp. 596-600, 2007.

[6] D. Zhao, C. Andreazza, P. Andreazza, J. Ma, Y. Liu, and D. Shen, "Buffer layer effect on ZnO nanorods growth alignment," Chemical Physics Letters, vol. 408, no. 4-6, pp. 335-338, 2005.

[7] J. Wang, Z. Wang, B. Huang, Y. Ma, Y. Liu, X. Qin, X. Zhang, and Y. Dai, "Oxygen Vacancy Induced Band-Gap Narrowing and Enhanced Visible Light Photocatalytic Activity of ZnO," ACS Applied Materials \& Interfaces, vol. 4, no. 8, pp. 4024-4030, 2012.

[8] N. Kaur, S. K. Sharma, and D. Young Kim, "Stress relaxation and transitions in optical bandgap of yttrium doped zinc oxide (YZO) thin films," Current Applied Physics, vol. 16, no. 3, pp. 231-239, 2016.

[9] S. Das, and T. L. Alford, "Optimization of the zinc oxide electron transport layer in P3HT:PC61BM based organic solar cells by annealing and yttrium doping," RSC Advances, vol. 5, no. 57, pp. 45586-45591, 2015.

[10] X. B. Li, Q. Q. Zhang, S. Y. Ma, G. X. Wan, F. M. Li, and X. L. Xu, "Microstructure optimization and gas sensing improvement of $\mathrm{ZnO}$ spherical structure through yttrium doping," Sensors and Actuators B: Chemical, vol. 195, pp. 526-533, 2014.

[11] Y. S. Bae, D. C. Kim, C. H. Ahn, J. H. Kim, and H. K. Cho, "Growth of ZnO nanorod arrays by hydrothermal method using homo-seed layers annealed at various temperatures," Surface and Interface Analysis, vol. 42, no. 6-7, pp. 978-982, 2010. 
[12] V. Kumar, V. Kumar, S. Som, A. Yousif, N. Singh, O. Ntwaeaborwa, A. Kapoor, and H. Swart, "Effect of annealing on the structural, morphological and photoluminescence properties of $\mathrm{ZnO}$ thin films prepared by spin coating," Journal of colloid and interface science, vol. 428, pp. 8-15, 2014.

[13] A. K. Singh, S. Chauhan, and R. Chandra, "Thickness dependent structural and magnetic properties of Nd2NiMnO6 epitaxial thin films," Thin Solid Films, vol. 625, pp. 17-23, 2017.

[14] S. K. Sharma, N. Kaur, J. Singh, A. Singh, P. Raj, S .Sankar, D. Y. Kim, N. Singh, N. Kaur, and H. Singh, "Salen decorated nanostructured $\mathrm{ZnO}$ chemosensor for the detection of mercuric ions (Hg2+)," Sensors and Actuators B: Chemical, vol. 232, pp. 712-721, 2016.

[15] P. K. Giri, S. Dhara, and R. Chakraborty, "Effect of $\mathrm{ZnO}$ seed layer on the catalytic growth of vertically aligned $\mathrm{ZnO}$ nanorod arrays," Materials Chemistry and Physics, vol. 122, no. 1, pp. 18-22, 2010.

[16] M. Su Kim, K. Gug Yim, H. Young Choi, M. Young Cho, G. Sik Kim, S. Min Jeon, D.-Y. Lee, J. Soo Kim, J. Su Kim, J.-S. Son, J. In Lee, and J.-Y. Leem, "Thermal annealing effects of MBE-seed-layers on properties of ZnO nanorods grown by hydrothermal method," Journal of Crystal Growth, vol. 326, no. 1, pp. 195-199, 2011.

[17] D.-T. Phan, and G.-S. Chung, "Effects of defects in Ga-doped ZnO nanorods formed by a hydrothermal method on CO sensing properties," Sensors and Actuators B: Chemical, vol. 187, pp. 191-197, 2013.

[18] H.-S. Choi, M. Vaseem, S. G. Kim, Y.-H. Im, and Y.-B. Hahn, "Growth of high aspect ratio ZnO nanorods by solution process: Effect of polyethyleneimine," Journal of Solid State Chemistry, vol. 189, pp. 25-31, 2012.

[19] C. Sui, Z. Lu, and T. Xu, "Effects of annealing temperature on photoluminescence of ZnO nanorods hydrothermally grown on a ZnO:Al seed layer," Optical Materials, vol. 35, no. 12, pp. 2649-2653, 2013.

[20] B. İkizler, , and S. M. Peker, "Effect of the seed layer thickness on the stability of ZnO nanorod arrays," Thin Solid Films, vol. 558, pp. 149-159, 2014.

[21] C.-Y. Kao, C.-L. Hsin, C.-W. Huang, S.-Y. Yu, C.-W. Wang, P.-H. Yeh, and W.-W. Wu, "High-yield synthesis of $\mathrm{ZnO}$ nanowire arrays and their opto-electrical properties," Nanoscale, vol. 4, no. 5, pp. 1476-1480, 2012. 